\title{
Methodenvergleich der Gesamteiweißbestimmung im Serum
}

\author{
Von P. DOERR und D. STAMM \\ Aus dem Max-Planck-Institut für Psychiatrie, München
}

(Eingegangen am 9. April 1968)

\section{Herrn Professor Scbütte in Dankbarkeit zum 60. Geburtstag gewidmet}

Am Beispiel der Serumgesamteiweißbestimmung wird ein allgemein anwendbares Modell entwickelt, das gestattet, Richtigkeit und Präzision von Methoden miteinander zu vergleichen, wenn diese eirie Größe bestimmen, die erst durch eine Bezugsmethode definiert ist.

Taking the determination of serum protein as an example, a general model is developed for comparing the accuracy and precision of methods when the measured value is defined by yet another method.

Die Ergebnisse klinisch-chemischer Analysen, die in verschiedenen Laboratorien mit verschiedenen Methoden gewonnen werden, lassen sich nur vergleichen, wenn mindestens noch zusätzlich folgende Informationen bekannt sind:

1. Die bei den Analysen der Proben im jeweiligen Labor aufgetretenen zufälligen und systematischen Fehler, die mit der statistischen Qualitätskontrolle abgeschätzt werden können (1).

2. Die Richtigkeit der benutzten Methode. Formal handelt es sich um den systematischen Fehler der Methode, der mit Hilfe der einfachen Regressionsanalyse ermittelt werden kann, falls man entweder Proben unterschiedlicher Konzentration mit bekanntem nahezu fehlerfreien Gehalt oder bekanntem Zusatz des Stoffes analysieren kann. Die Ermittlung des systematischen Fehlers einer Methode wird komplizierter, wenn diese Methode eine Größe bestimmt, die erst durch eine konventionell festgelegte Standardmethode definiert ist. Die mit der Standardmethode (Bezugsmethode) durchgeführten Bestimmungen sind bei Abwesenheit laboreigener systematischer Fehler zwar „richtig“, jedoch haftet ihnen häufig ein nicht vernachlässigbar kleiner Zufallsfehler an.

Am Beispiel der Serumgesamteiweißbestimmung soll gezeigt werden, wie die genannten Parameter erfaßt werden können. Dabei wurde als Standardmethode die KjeldahlMethode mit der Biuret-Methode, der Spezifischen Gewichtsmethode nach VAN SLYKE und der Refraktometrie verglichen.

$\mathrm{Da}$ Serumeiweiß ein vielfältiges Gemisch von Proteinen variabler Aminosäurenzusammensetzung mit unterschiedlichem Gehalt an Lipiden, Kohlenhydraten und anderen Begleitbestandteilen darstellt, ist es nicht verwunderlich, daß jede der vier hier betrachteten Methoden eine andere physikalische oder chemische Eigenschaft mißt, die als mehr oder weniger charakteristisch für dieses Proteingemisch angesehen werden kann. Die konventionelle Angabe von Gesamteiweiß in $\mathrm{g} / 100 \mathrm{ml}$ täuscht eine zuverlässige gravimetrische Bezugsmethode vor. In Abhängigkeit von sehr speziellen Bedingungen bei der präparativen Darstellung der Proteine (Dialyse, Extraktion und Trocknungsverfahren) ergeben sich unterschiedliche Resultate $(2,3)$. Es erscheint daher auch heute noch zweckmäßig, die Protein-N-Konzentration als $\mathrm{Maß}$ für die Gesamteiweißkonzentration im
Serum zu benützen: Serumgesamteiweiß $(\mathrm{g} / 100 \mathrm{~m} /)=$ $\mathrm{k} \cdot$ Protein-N $(\mathrm{g} / 100 \mathrm{~m} /)$. Die für den Proportionalitätsfaktor $\mathrm{k}$ angegebenen Zahlenwerte sind abhängig vom zugrunde gelegten Verfahren der gravimetrischen Proteinbestimmung. Wir benutzen daher in dieser Arbeit weiterhin den konventionellen Faktor 6, 25. Sachlich am konsequentesten erschiene es uns, so lange Serumgesamteiweiß in $\mathrm{g}$ Protein-N/100 $\mathrm{m} l$ anzugeben, als man den Stickstoffgehalt des Proteingemisches für die optimale Bezugsgröße hält.

\section{Methoden der Serumgesamteiweißbestimmung}

\section{Kjeldahl-Methode}

Es wurde die in den Standard-Methods of Clinical Chemistry (4) beschriebene Mikro-Kjeldahl-Methode benutzt, die auf der von Hiller, Plazin und v. Siyke (5) ausgearbeiteten Methode beruht. Lediglich die Titration der unverbrauchten Schwefelsäure wurde mit dem Titrator TTT $1 \mathbf{c}^{1}$ ) durchgeführt. Dabei kann der Endpunkt der Titration genauer erfaßt werden, da er durch den gemessenen $\mathrm{pH}$-Wert bestimmt wird. Außer dem Gesamt-N des Serums wurde noch der Rest-N nach der von v. SLYKE angegebenen Beziehung: Rest-N $=10+1,07 \cdot$ Harnstoff-N berechnet. Der Harnstoff-N wurde mit der Urease-Methode (6) bestimmt. $\mathrm{Da}$ bei Normalseren der Rest-N nur 2-3\% des Total-N ausmacht, ist der Fehler der Protein-N-Bestimmung unter dieser Voraussetzung fast restlos durch die Kjeldahl-Methode bedingt.

\section{Biuret-Methode}

Die in den Standard-Methods (7) angegebene Methode nach Weichselbaum (8) wurde ohne wesentliche Änderung übernommen: Wir benutzten $10 \mu l$ Serum, die mit einer MarburgPipette $^{2}$ ) pipettiert wurden.

Als Standardprobe diente der serumähnliche Eiweißstandard $\mathrm{Labtro}^{3}$ ), dessen Eiweißgehalt mit der Kjeldahl-Methode bestimmt ist.

\section{Spezifische Gewichtsmethode}

Es wurde die von $\nabla$. SLYKKE und Mitarbeitern $(9,10)$ ausgearbeitete Methode übernommen, bei der das spezifische Gewicht des Serums durch den Vergleich mit den spezifischen Gewichten einer Verdünnungsreihe einer Kupfersulfatlösung bestimmt wird. Die von v. SLYKE angegebene Analyseneichfunktion lautete:

$$
\mathrm{P}=369 \cdot(\mathrm{G}-\mathrm{b})
$$

$\mathrm{P}$ : Serumgesamteiwei $\beta$ in $\mathrm{g} / 100 \mathrm{ml}$

G: spezifisches Gewicht der Serumprobe $(\mathrm{g} / \mathrm{m} /)$

b: spezifisches Gewicht der im Serum gelösten Nichteiweißbestandteile, das als Konstante mit dem Wert $\mathrm{b}=1,007 \mathrm{~g} / \mathrm{ml}$ aufgefaßt wird.

1) Hersteller: Radiometer, Kopenhagen.

2) Hersteller: Eppendorf Gerätebau, Netheler \& Hinz GmbH, Hamburg.

3) Hersteller: DADE Reagents, Ltd. Miami, USA. 
Refraktometrische Serumgesamteiweißbestimmung

Wir benutzten im Prinzip die von ReIss 1902 in die Klinik eingeführte Methode $(11,12)$. Er setzte voraus, da $B$ der Brechungsindex des Serums sich additiv aus dem Brechungsindex des gelösten Serumeiweißes und der Nichteiweißbestandteile zusammensetzte, und nahm an, daß der Brechungsindex der Nichteiweißbestandteile als konstant angesehen werden könne. Die von ihm angegebenen Zahlenwerte für den Brechungsindex einer 1 proz. Serumeiweißlösung und den Brechungsindex der Nichteiweißkörper wurden später wiederholt korrigiert.

Wir verwandten die von ALDER (13) angegebenen Konstanten mit der Temperaturkorrektur von LOEWE (14). Zur Bestimmung des Brechungsindex des Serums benutzten wir das Abbe-Refraktometer Modell A der Firma Carl Zeiss mit einer Temperierung der Prismen im Durchfluß auf $20^{\circ}\left( \pm 0,1^{\circ}\right)$. Bei jeder Probenmessung wurde die Grenzlinie des Hell-Dunkelfeldes dreimal auf das Strichkreuz eingestellt. Das arithmetische Mittel der drei abgelesenen Brechungsindices wird als Brechungsindex der Probe angesehen.
2. Es wurden keine Proben benutzt, deren Bestandteile als Störfaktoren mit Sicherheit bekannt und selbst überprüft waren. Lipämie stört bei der Biuret-Methode und der Refraktometrie, gering auch bei der spezifischen Gewichtsmethode. Erhöhte Konzentrationen von Harnstoff und Glucose stören bei der Refraktometrie und der Spezifischen Gewichtsmethode $(10,12$, 13, 14).

Konzentrationsbereich und Häufigkeit der erfaßten pathologischen Serumbestandteile von 50 Serumproben sind in Tabelle $2 \mathrm{zu}-$ sammengefaßt.

\section{Statistische Auswertungen der Meßergebnisse}

$\mathrm{Da}$ sich bis jetzt eine hinreichend einheitliche Nomenklatur in der Statistik nicht durchgesetzt hat, werden im Folgenden sämtliche Formeln zur Errechnung der Parameter und Testgrößen angegeben, die zur Beurteilung der Meßergebnisse benutzt wurden. Zugleich wird damit zur Diskussion gestellt, inwieweit der Auswahl und Anwendung statistischer Prüfverfahren auf das Zahlenmaterial subjektive Gesichtspunkte zugrunde liegen.

Versuchsanordnung

Tab. 1

Art und Anzahl der täglich innerhalb eines Zeitraumes von 1 Monat analysierten Proben

\begin{tabular}{|c|c|c|c|c|}
\hline Methode & Pathotrol & Monitrol I & normale Seren & path. Seren \\
\hline Kjeldahl & 1 Doppelbest. & 1 Doppelbest. & $\begin{array}{l}1 \text { Doppelbest. und } \\
1 \text { oder } 2 \text { Einfachbest. }\end{array}$ & 2 oder 3 Einfachbest. \\
\hline Biuret & - & 1 Doppelbest. & $\begin{array}{l}1 \text { Doppelbest. und } \\
1 \text { oder } 2 \text { Einfachbest. }\end{array}$ & 2 oder 3 Einfachbest. \\
\hline Spez. Gew. & - & 1 Doppelbest. & $\begin{array}{l}1 \text { Doppelbst. und } \\
1 \text { oder } 2 \text { Einfachbest. }\end{array}$ & 2 oder 3 Einfachbest. \\
\hline Refraktometrie & - & 1 Doppelbest. & $\begin{array}{l}1 \text { Doppelbest. und } \\
1 \text { oder } 2 \text { Einfachbest. }\end{array}$ & 2 oder 3 Einfachbest. \\
\hline
\end{tabular}

Aus Tabelle 1 sind Art und Anzahl der täglichen in einem Zeitraum von einem Monat mit den vier beschriebenen Methoden analysierten Proben ersichtlich. Die Richtigkeit der Analysen nach der Kjeldahl-Methode, die bei dieser Versuchsanordnung als Bezugsmethode dient, wird mit dem lyophilisierten Normalserum Monitrol $\mathrm{I}^{4}$ ), dessen Bestandteile in mehreren voneinander unabhängigen Laboratorien in vielen Einzelanalysen bestimmt werden, und dem serumähnlichen Eiweißstandard Pathotrol ${ }^{4}$ ) überwacht. Die Doppelbestimmungen aus den Normalseren und Monitrol I dienen zur Ermittlung der Präzision in der Serie [1], zusätzlich läßt sich aus den Monitrol-Bestimmungen für aufeinanderfolgende Tage die Präzision von Tag zu Tag berechnen [2]. $\mathrm{Da}$ ein und dieselbe Probe mit vier verschiedenen Methoden bestimmt wird, kann einem Kjeldahl-Wert je eine Bestimmung der anderen 3 Methoden zugeordnet werden. Bei der Auswahl der pathologischen Seren wurden folgende Gesichtspunkte berücksichtigt

1. Die Gesamteiweißkonzentrationen der Proben sollten einen möglichst weiten Konzentrationsbereich erfassen.

Tab. 2

Zusammensetzung der pathologischen Seren, $\mathbf{n}=\mathbf{5 0}$

\begin{tabular}{lcc}
\hline \multicolumn{1}{c}{ Serumbestandteil } & Bereich & Anzahl \\
\hline Bilirubin, gesamt $(\mathrm{mg} / 100 \mathrm{ml})$ & $1,1-18,4$ & 15 \\
Harnstoff-N $(\mathrm{mg} / 100 \mathrm{ml})$ & $\mathbf{2 2 - 8 2 , 3}$ & 20 \\
Gesamt-EiweiB $\left(\mathrm{g} / 100 \mathrm{~m}{ }^{\prime}\right)$ & $3,6-\mathbf{6 , 0}$ & 22 \\
& $\mathbf{8 , 0 - 1 1 , 9}$ & 16 \\
Albumin rel. $\%$ & $36-55$ & 20 \\
$\alpha_{1}$-Globuline & $4,5-11,0$ & 5 \\
$\alpha_{2}$-Globuline & $9,5-21,0$ & 21 \\
$\beta$-Globuline & $12,6-18,0$ & 6 \\
$\gamma$-Globuline & $22,2-55,0$ & 25
\end{tabular}
Keine lipämischen Seren, Harnstoff-N nicht über $82,3 \mathrm{mg} / 100 \mathrm{ml}$, fette Ziffern.

4) Herstellex: DADE Reagents „Ltd. Miami USA.
Präzision in der Serie aus Doppelbestimmungen:

$$
s=\sqrt{\frac{\Sigma\left(x_{1_{1}}-x_{i_{2}}\right)^{2}}{2 m}}
$$

m: Anzahl der Doppelbestimmungen

$\mathbf{x}_{1_{1}}, \mathbf{x}_{\mathbf{i}_{2}}$ : eine Doppelbestimmung

$$
\text { mit } \mathrm{f}=\mathrm{m} \text { Freiheitsgraden }
$$

Präzision von Tag zu Tag:

$\mathrm{n}$ : Anzahl der Meßwerte $\mathbf{x}_{\mathbf{i}}$

$\overline{\mathbf{X}}$ : arithm. Mittel der Meßwerte

$$
\mathrm{s}=\sqrt{\frac{\Sigma\left(\mathrm{x}_{1}-\overline{\mathrm{x}}\right)^{2}}{\mathrm{n}-1}}
$$

mit $f=n-1$ Freiheitsgraden

Variationskoeffizient:

$$
\begin{aligned}
& V=\frac{s \cdot 100}{\bar{x}} \\
& s_{0}=s \sqrt{F\left(P / 2, f_{1}=\infty, f_{2}=n-1\right)}
\end{aligned}
$$

$s_{0}$ : obere Grenze

$s_{u}$ : untere Grenze

$f:$ Freiheitsgrade von $s$

F: Integralgrenze der F-Verteilung in Abhängigkeit der Freiheitsgrade $f_{1}$ und $f_{2}$ für die vorgegebene statistische Sicherheit $P$

$$
s_{u}=s \sqrt{\frac{1}{F\left(P / 2, f_{1}=n-1, f_{2}=\infty\right)}}
$$

$t=$ Test zum Nachweis eines systematischen Fehlers bei nfacher Bestimmung einer Probe mit dem bekannten Gehalt $\mu$

Falls $t>t(P, f)$, ist $|\mu-\bar{x}|$ signifikant 
F-Test zur Entscheidung, ob die beiden Varianzen $s_{1}^{2}$ und $s_{2}^{2}\left(s_{1}>s_{2}\right)$ Schätzwerte derselben Varianz $\sigma^{2}$ sein können.

$$
\mathrm{F}=\frac{\mathrm{s}_{1}^{2}}{\mathrm{~s}_{2}^{2}}
$$

mit den Freiheitsgraden $f_{1}=n_{1}-1$ und $f_{2}=n_{2}-1$

Falls $F>F\left(P / 2, f_{1}, f_{2}\right)$, ist die

Annahme, $s_{1}^{2}$ und $s_{2}^{2}$ seien

Schätzwerte derselben Varianz

$\sigma^{2}$, zu verwerfen.

Bei der Berechnung der Regressionsgleichung

$$
y=a+b x
$$

werden die Kjeldahl-Werte der Normalseren $x_{K \mathbf{j} 1}$ und der pathologischen Seren $\mathbf{x}_{\mathbf{K} 2}$ als unabhängige Variable aufgefaßt. Jedem Meßwert einer Kjeldahl-Bestimmung $x_{i}$ werden die entsprechenden $y_{i}$-Werte der drei anderen Methoden zugeordnet. Die KjeldahlWerte sind ausgesucht unter dem Gesichtspunkt einer möglichst gleichmäßigen Erfassung der gesamten tatsächlich vorkommenden Serumgesamteiweißkonzentrationen. Den Kjeldahl-Werten liegt zwar eine Zufallsverteilung zugrunde, sie entspricht aber nicht einer Zufallsstichprobe aus einer Grundgesamtheit. Für das Folgende betrachten wir die Kjeldahl-Werte als fest vorgegeben. Sämtlichen Einzelwerten haften zufällige Fehler an, für die eine Normalverteilung mit derselben Varianz $\sigma^{2}$ vorausgesetzt wird. Die Annahme, daß y $=f(x)$ linear ist, läßt sich für das vorliegende Zahlenmaterial mit Hilfe der Varianzanalyse nicht testen, da erstens einem Kjeldahl-Wert nur je ein Wert der drei anderen Methoden zugeordnet werden kann und zweitens die Zufallsfehler der Kjeldahl-Werte nicht als vernachlässigbar klein angesehen werden können. Es wurde statt dessen der Korrelationskoeffizient $r$ [11] berechnet, der ein $\mathrm{Maß}$ für die gegenseitige Abhängigkeit zweier zufallsverteilter Größen darstellt, aber unabhängig von der Eigenschaft der Zufälligkeit auch eine Maßzahl für die Linearisierbarkeit des funktionalen Zusammenhanges zwischen zwei Größen ist.

Für jede Regressionsgleichung wurde nach [13] getestet, ob der Ordinatenabschnitt a von Null signifikant verschieden ist. Die Annahme, a sei gleich Null, wird für unser Zahlenmaterial verworfen, wenn $|t|>t(P=0,05, f)$ ist. Der Regressionskoeffizient $\mathrm{b}^{\prime}$ wird nach [17] getestet auf seinen signifikanten Unterschied von 1. Die Hypothese, $b^{\prime}$ sei gleich 1 , wird verworfen, wenn $|t|>t(P=0,01, f)$ ist. In diesem Fall liegt ein systematischer Unterschied zwischen den Werten der Bezugsmethode (KjeldahlMethode) und den Werten der betreffenden Vergleichsmethode vor. Falls die Analysen mit der Kjeldahl-Methode frei von systematischen Fehlern sind, was mit den Kontrollproben Monitrol I und Pathotrol nach dem angegebenen Testkriterium [5] geprüft wird, kann der aufgetretene systematische Unterschied nur noch bedingt sein durch a) einen laboreigenen systematischen Fehler bei der Durchführung der Analysen mit der Vergleichsmethode, b) durch einen systematischen Unterschied $z$ wischen der KjeldahlMethode und der Vergleichsmethode oder aber durch die unter a) und b) genannten Ursachen zugleich. Die Kenntnis des Regressionskoeffizienten $b^{\prime}$ etlaubt in einfacher Weise die Umrechnung des Eiweißgehaltes einer Probe, der nach der KjeldahlMethode bestimmt ist, in den zu erwartenden Eiweißgehalt der Probe, falls er mit der Vergleichsmethode bestimmt wird. Diese Umrechnung ist zunächst nur zulässig für das Labor, in dem die Regressionsanalyse durchgeführt wurde, da über die Berechtigung der Annahme, der laboreigene systematische Fehler der Analysen der Vergleichsmethode sei vernachlässigbar klein gegenüber dem systematischen Unterschied zwischen der Kjeldahl-Methode und der Vergleichsmethode, nichts bekannt ist. Erst wenn in mehreren voneinander unabhängigen Laboratorien die gleichen Regressionsanalysen durchgeführt sind, ist eine Aussage über den systematischen Unterschied der betrachteten Eiweißbestimmungsmethoden gegenüber der Kjeldahl-Methode möglich.
Insgesamt wurden folgende Parameter und Testgrößen berechnet $(16,17,18)$ :

Regressionskeeffizient: $\quad \mathrm{b}=\frac{\mathrm{n} \Sigma \mathrm{x}_{1} \mathrm{y}_{1}-\Sigma \mathrm{x}_{1} \Sigma_{\mathrm{y}_{1}}}{\mathrm{n} \Sigma \mathrm{x}_{1}^{2}-\left(\Sigma \mathrm{x}_{1}\right)^{2}}$

additiv konstantes Glied: $\quad \mathrm{a}=\overline{\mathrm{y}}-\mathrm{b} \overline{\mathrm{x}}$

$\begin{aligned} & \text { Streuung der } \mathrm{y}_{\mathrm{i}} \text {-Werte um die } \\ & \text { Regressionsgerade: }\end{aligned} \mathrm{s}_{\mathrm{yx}}=\sqrt{\frac{\Sigma\left(\mathrm{y}_{\mathrm{i}}-\mathrm{Y}_{\mathrm{i}}\right)^{2}}{\mathrm{n}-2}}$

$$
\begin{gathered}
\Sigma\left(\mathrm{y}_{\mathrm{i}}-\mathrm{Y}_{\mathrm{i}}\right)^{2}=\Sigma_{\mathrm{y}_{1}^{2}-\mathrm{ny} \overline{\mathrm{y}}^{2}} \\
-\mathrm{b}^{2}\left(\Sigma \mathrm{x}_{\mathrm{i}}^{2}-\mathrm{n} \overline{\mathrm{x}}^{2}\right)
\end{gathered}
$$

mit $f=n-2$ Freiheitsgraden

Korrelationskoeffizient: $\quad \mathrm{I}=\frac{\mathrm{n} \Sigma_{\mathrm{x}_{1}} \mathrm{y}_{\mathrm{i}}-\Sigma \mathrm{x}_{\mathrm{i}} \Sigma_{\mathrm{y}_{\mathrm{i}}}}{\sqrt{\left[\mathrm{n} \Sigma \mathrm{x}_{\mathrm{i}}^{2}-\left(\Sigma \mathrm{x}_{\mathrm{i}}\right)^{2}\right]\left[\mathrm{n} \Sigma_{\mathrm{y}_{1}}-\left(\Sigma \mathrm{y}_{\mathrm{i}}\right)^{2}\right]}}$

$t$-Test für signifikanten Unterschied $\mathrm{r}$ von 0

$$
\mathrm{t}=\frac{\mathrm{r}}{\sqrt{1-\mathrm{r}^{2}}} \sqrt{\mathrm{n}-2}
$$

mit $f=n-2$ Freiheitsgraden

t-Test für signifikanten Unterschied a von 0

$$
t=-\frac{a}{s_{y x} \sqrt{\frac{1}{n}+\frac{\bar{x}^{2}}{\Sigma\left(x_{i}-\bar{x}\right)^{2}}}}
$$

$$
\text { mit } f=n-2 \text { Freiheitsgraden }
$$

Falls a von 0 nicht signifikant verschieden ist, wird $a=0$ gesetzt und die neue Regressionsgerade berechnet:

$$
\begin{array}{cc}
\mathrm{y} & =\mathrm{b}^{\prime} \mathrm{x} \\
\text { Anstieg der Regressionsgeraden } & \mathrm{b}^{\prime}=\frac{\Sigma \mathrm{x}_{\mathrm{i}} \mathrm{y}_{\mathrm{i}}}{\Sigma \mathrm{x}^{\prime}}
\end{array}
$$

Streutung der $\mathrm{y}_{i}$-Werte um die Regressionsgerade [14]

$\mathrm{s}_{\mathrm{yx}}^{\prime}=\sqrt{\frac{\sum\left(\mathrm{y}_{\mathrm{i}}-\mathrm{b}^{\prime} \mathrm{x}_{\mathrm{i}}\right)^{2}}{\mathrm{n}-1}}$

mit $f=n-1$ Freiheitsgraden

$t$-Test für signifikanten Unterschied $b^{\prime}$ von 1

$$
\mathrm{t}=\frac{1-\mathrm{b}^{\prime}}{\mathrm{s}^{\prime} \mathrm{yx}} \cdot \sqrt{\Sigma \mathrm{x}_{\mathrm{i}}^{2}}
$$

mit $f=n-1$ Freiheitsgraden

\section{Ergebnisse und Diskussion}

Tab. 3

Präzision in der Serie für Normalseren Vertrauensintervall von $s$ für $P=0,05, m=20$ s. [1], [3] und [4]

\begin{tabular}{lcccc}
\hline \multicolumn{1}{c}{ Methode } & $\mathrm{s}$ & $\mathrm{s}_{\mathbf{u}}$ & $\mathrm{s}_{\mathbf{o}}$ & $\mathrm{V}$ \\
\hline Kjeldahl & 0,115 & 0,092 & 0,156 & 1,5 \\
Biuret & 0,130 & 0,104 & 0,177 & 1,8 \\
Spez. Gew. & 0,106 & 0,085 & 0,144 & 1,5 \\
Refraktometrie & 0,034 & 0,027 & 0,046 & 0,5 \\
\hline
\end{tabular}

Tab. 4

Präzision in der Serie für Monitrol I

Vertrauensintervall von $s$ für $P=0,05, m=20$ s. [1], [3] und [4]

\begin{tabular}{lcccc}
\hline \multicolumn{1}{c}{ Methode } & $\mathrm{s}$ & $\mathrm{s}_{\mathbf{u}}$ & $\mathrm{s}_{\mathbf{0}}$ & $\mathrm{V}$ \\
\hline Kjeldahl & 0,137 & 0,110 & 0,186 & 1,9 \\
Biuret & 0,137 & 0,110 & 0,186 & 1,9 \\
Spez. Gew. & 0,084 & 0,067 & 0,114 & 1,2 \\
Refraktometrie & 0,040 & 0,032 & 0,054 & 0,6, \\
\hline
\end{tabular}


Tab. 5

Präzision von Tag zu Tag für Monitrol I Vertrauensintervall von $s$ für $P=0,05, n=20$ s. [2], [3] und [4]

\begin{tabular}{lcccc}
\hline \multicolumn{1}{c}{ Methode } & $\mathrm{s}$ & $\mathrm{s}_{\mathrm{u}}$ & $\mathrm{s}_{\mathrm{o}}$ & $\mathrm{V}$ \\
\hline Kjeldahl & 0,167 & $\mathbf{0 , 1 3 2}$ & $\mathbf{0 , 2 2 6}$ & $\mathbf{2 , 3}$ \\
Biuret & 0,176 & 0,139 & $\mathbf{0 , 2 3 8}$ & $\mathbf{2 , 5}$ \\
Spez. Gew. & 0,084 & 0,066 & 0,113 & $\mathbf{1 , 2}$ \\
Refraktometrie & 0,067 & 0,053 & 0,091 & 0,9 \\
\hline
\end{tabular}

Tab. 6

Richtigkeitskontrolle der Kjeldahl-Analysen $n=20, t(P=0,05, f=19)=2,09$, s. [5] Eiweißgehalt in $g / 100 \mathrm{ml}$

\begin{tabular}{cccc}
\hline Kontrollprobe & $\boldsymbol{\mu}$ & $\overline{\mathbf{x}}$ & $\mathrm{t}$ \\
\hline Monitrol I & 7,10 & $\mathbf{7 , 1 3}$ & 0,80 \\
Pathotrol & 5,00 & 5,03 & 1,09 \\
\hline
\end{tabular}

Tab. 7

Abhängigkeit der Serumgesamteiweißbestimmungen nach der Biuret-Methode, der Spezifischen Gewichtsmethode und der Refraktometrie von den Kjeldahl-Bestimmungen an 50 Normalseren. Konzentrationsbereich $6,0-8,0 \mathrm{~g} / 100 \mathrm{ml}$

\begin{tabular}{|c|c|c|c|}
\hline Berechnung nach & Biuret-Methode & Spez. Gew.-Methode & Refraktometrie \\
\hline $\begin{array}{l}{[7],[8],[9]} \\
{[10]} \\
{[11]} \\
{[12]} \\
{[13]} \\
{[14],[15]} \\
{[16]} \\
{[17]}\end{array}$ & $\begin{aligned} \mathbf{y}_{\mathbf{B i}} & =1,00+0,82 x_{K \mathbf{j}} \\
\mathbf{s}_{\mathbf{y x}} & =0,31 \\
\mathbf{r} & =0,80 \\
t & =9,29 \\
t & =0,83 \\
\mathbf{y}_{\mathbf{B i}}^{\prime} & =0,96 \times_{\mathbf{K} j} \\
s_{\mathbf{y x}}^{\prime} & =0,31 \\
t & =6,55\end{aligned}$ & $\begin{aligned} \mathbf{y}_{\mathbf{S G}} & =0,93+0,90 \mathbf{x}_{\mathbf{K j}} \\
\mathbf{s}_{\mathbf{y x}} & =0,25 \\
\mathbf{r} & =0,88 \\
\mathbf{t} & =12,5 \\
\mathbf{t} & =0,96 \\
\mathbf{y}_{\mathbf{S G}} & =1,03 \mathbf{x}_{\mathbf{K j}} \\
\mathbf{s}_{\mathbf{y x}}^{\prime} & =0,25 \\
\mathbf{t} & =6,06\end{aligned}$ & $\begin{aligned} \mathrm{y}_{\mathbf{R}} & =1,65+0,79 \mathrm{x}_{\mathbf{K} \mathbf{j}} \\
\mathbf{s}_{\mathbf{x y}} & =0,29 \\
\mathrm{r} & =0,81 \\
\mathrm{t} & =9,67 \\
\mathrm{t} & =1,48 \\
\mathbf{y}_{\mathbf{R}}^{\prime} & =1,03 \mathrm{x}_{\mathbf{K} \mathbf{j}} \\
\mathrm{s}_{\mathbf{y} \mathbf{x}}^{\prime} & =0,31 \\
\mathrm{t} & =5,05\end{aligned}$ \\
\hline
\end{tabular}

Tab. 8

Abhängigkeit der Serumgesamteiweißbestimmungen nach der Biuret-Methode, der Spezifischen Gewichtsmethode und der Refraktometrie von den Kjeldahl-Bestimmungen an 50 pathologischen Seren. Konzentrationsbereich $3,6-11,9 \mathrm{~g} / 100 \mathrm{ml}$

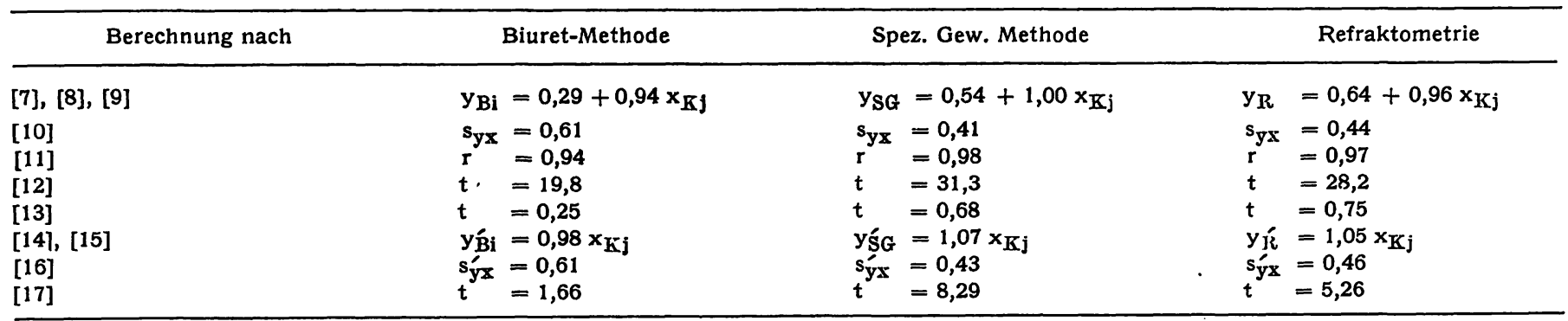
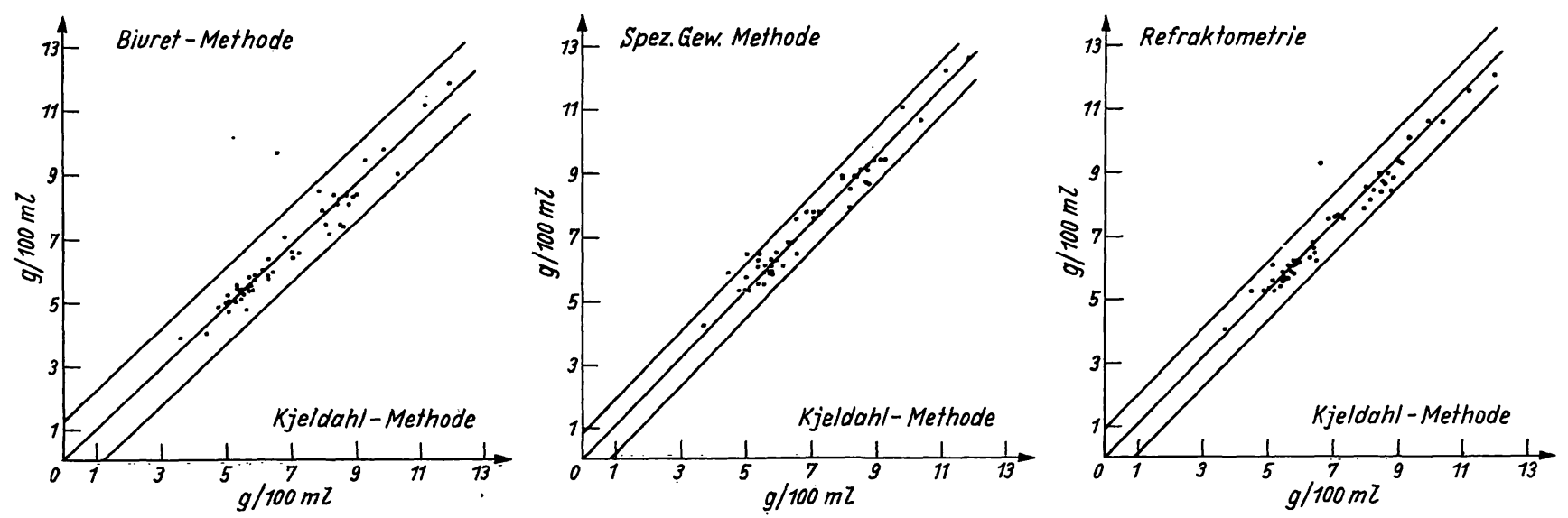

Abb. $1-3$

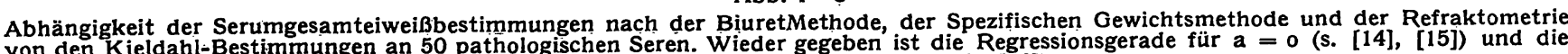
von den Kjeldahl-Bestimmungen an 50 pathologischen Seren. Wieder gegeben ist die Regression
doppelte Standardabweichung $\pm 2 s^{\prime}$ yx (s. [16])

Die Präzision in der Serie und die Präzision von Tag zu Tag, die von uns für die vier verschiedenen Methoden der Serumeiweißbestimmung gefunden wurden, sind in den Tabellen 3, 4 und 5 wiedergegeben. Es fällt auf, $\mathrm{da} \beta$ die Refraktometrie die höchste Präzision besitzt. Die Biuret- und Kjeldahl-Methode unterscheiden sich nicht, eine $Z$ wischenstellung nimmt die Spezifische Gewichtsmethode ein. Statistisch signifikant $(P<0,01)$ ist lediglich der Unterschied der Präzision in der Serie gegenüber der Präzision von Tag zu Tag für die Refraktometrie. Die relativ geringe Präzision der BiuretMethode ist vorwiegend durch die Volumenmessung der $10 \mu l$ bedingt und ließe sich bei Verwendung eines geeigneten Dilutors oder durch Vergrößerung der Probemenge auf 50-100 $\mu l$ verbessern.

Aus Tabelle 6 geht hervor, daß die mit der KjedahlMethode durchgeführten Analysen frei von systematischen Fehlern waren, denn die nach [5] berechneten 
t-Werte zeigen, daß die arithmetischen Mittel der Kontrollproben vom vorgegebenen richtigen Wert nicht signifikant verschieden sind.

Die Tabelle 7 und 8 gibt die für das experimentelle Zahlenmaterial berechneten Regressionsgleichungen und Testgrößen wieder, deren formale Bedeutung im vorigen Abschnitt erörtert wurde. Es fällt auf, daß kein Ordinatenabschnitt von 0 signifikant verschieden ist [13]. In jedem Fall konnte die Regressionsgleichung für $\mathrm{a}=0$ berechnet werden $[14,15]$.

Der Regressionskoeffizient $b^{\prime}$ [15] ist bei der BiuretMethode für die Normalseren und die pathologischen Seren kleiner als 1, die Abweichung von 1 ist aber nur bei den Normalseren statistisch signifikant [17].

Unserer Meinung nach kann daraus der Schluß gezogen werden, daß bei den mit der Biuret-Methode durchgeführten Analysen ein systematischer Fehler von etwa $2-4 \%$ aufgetreten ist. Ein systematischer Unterschied zwischen der Biuret-Methode und der Kjeldahl-Methode kommt nicht in Betracht, da der Eiweißgehalt der Standardprobe bei der von uns benutzten Biuret-Methode mit der Kjeldahl-Methode bestimmt ist.

Bei der Spezifischen Gewichtsmethode sind die Regressionskoeffizienten $b^{\prime}$ größer als 1. Formal läßt sich nicht unterscheiden, ob dies durch einen laboreigenen systematischen Fehler der Analysen der Vergleichmethode oder durch einen systematischen Unterschied zwischen der Vergleichsmethode und der Bezugsmethode bedingt ist. In diesem Zusammenhang erscheint es bemerkenswert, daß Heusser und SCHNEIDER (19) bei der Überprüfung der Analyseneichfunktion einen niedrigeren Proportionalitätsfaktor $(\mathrm{k}=360)$ gefunden haben als v. SLYKe $(k=369)$. Bei einem Wert von $k=358$ würde bei den Normalseren kein Unterschied zwischen den nach der Kjeldahl-Methode und den nach der Spezifischen Gewichtsmethode bestimmten Eiweißwerten bestehen. Bei den pathologischen Seren bliebe jedoch auch dann noch ein von 1 signifikanter Unterschied, der wahrscheinlich auf die Anwesenheit von Störfaktoren zurückzuführen ist. Eine sichere Zuordnung dieser geforderten Störfaktoren zu den von uns erfaßten pathologischen Serumbestandteilen war nicht möglich.

Ähnlich wie bei der spezifischen Gewichtsmethode liegen auch die Verhältnisse bei der Refraktometrie. Die Regressionskoeffizienten $b^{\prime}$ sind ebenfalls signifikant größer als 1. Die refraktometrisch bestimmten Eiweißwerte liegen bei den Normalseren im Durchschnitt um $3 \%$, bei den pathologischen Seren um 5\% höher als die Kjeldahl-Werte.
Welcher Anteil dieses systematischen Fehlers auf einen laboreigenen systematischen Fehler bei den durchgeführten Analysen der Refraktometrie und welcher Anteil auf den systematischen Fehler der Vergleichsmethode selbst zurückzuführen ist, läßt sich nicht unterscheiden. Es wäre daher verfrüht, den Proportionalitätsfaktor der Anälyseneichfunktion zu korrigieren. Für unser Labor ist es möglich, den Eiweißgehalt eines Kontrollserums für die Refraktometrie oder die spezifische Gewichtsmethode zu berechnen, was die Voraussetzung für eine komplette Qualitätskontrolle ist.

Bei der Betrachtung der Korrelationskoeffizienten fällt auf, $\mathrm{da} ß$ diese bei den pathologischen Seren größer sind als bei den Normalseren. Dies kann möglicherweise so gedeutet werden, daß für das vorliegende Zahlenmaterial der Zusammenhang zwar nicht exakt linear ist, sich aber über einen größeren Bereich, wie er bei den pathologischen Seren vorliegt, im Mittel gut linearisieren läßt. Mit Hilfe der pathologischen Seren konnte einerseits gezeigt werden, daß sich der Zusammenhang zwischen den Werten der Bezugsmethode und den Werten der Vergleichmethode gut linearisieren läßt, zum anderen, $\mathrm{da} B$ systematische Fehler durch bisher nicht bekannte Störfaktoren nur gering sein können und nur bei der spezifischen Gewichtsmethode und der Refraktometrie in Erwägung gezogen werden müssen.

Der Wert der spezifischen Gewichtsmethode und der Refraktometrie ist trotz der hohen Präzision dieser Methoden erheblich dadurch eingeschränkt, daß ohne gleichzeitige Kenntnis der Glucose- und Harnstoffkonzentrationen einer Probe der bestimmte Eiweißgehalt keine Aussagekraft besitzt. Wenn es daher fraglich erscheinen sollte, ob der hier betriebene Aufwand berechtigt ist, so möchten wir betonen, daß der Vergleich der Serumeiweißbestimmungsmethoden vor allem ein Beispiel dafür sein soll, auf welche Weise und mit welchen statistischen Hilfsmitteln Richtigkeit und Präzision von Methoden miteinander verglichen werden können, falls sie eine Größe bestimmen, die erst durch eine Bezugsmethode definiert ist.

Herrn Dr. E. HANSERT, Leiter unserer Biometrischen Abteilung, danken wir ganz herzlich für wichtige Anregungen und wertvolle Diskussionen bei der Planung und Durchführung der Arbeit.

Herrn Dr. Oberdorfer, Chefarzt des Klinisch Chemischen Instituts des Klinikums rechts der Isar der Technischen Hochschule München, danken wir für die liebenswürdige UUberlassung von Serumproben.

\section{Literatur}

1. Büttner, H., diese Z. 5, 41 (1967). - 2. Armstrong, S. H. jr., M. J. E. Budka, K. C. Morrison und M. Hasson; J. Amer. chem. Soc., 69, 1747 (1947). - 3. SundermanN, F. W. jr., F. W. Sundermand, E. A. Flavo, C. J. Kallick, Amer. Clin. Path. 30, 112 (1958). - 4. Archibald, R. M., Standard Methods of Clinical Chemistry, Vol. II, S. 91, Academic Press Inc., Publishers New York (1958). - 5. Hiller, A., J. Plazin und D. D. v. Slyke, J. biol. Chemistry 176, 1401 (1948). - 6. HENRY, R. J., Clinical Chemistry, 1. Aufl., S. 266, Hoeber Medical Division, Harper \&
Row, Publishers, New York (1964). - 7. Reinhold, J. G., Standards Methods of Clinical Chemistry Vol. I, S. 88, Academic Press Inc., Publishers, New York (1953). - 8. Weichsenbaum, T. E. Amer. J. Clin. Path. 7, 40 (1946). - 9. Philliss, R. A., D. D. v. Slyke, P. B. Hamilton, V. P. Dole, K. Emerson jr., R. M. Archibald, J. biol. Chemistry 183, 305 (1950). - 10.v. SlyKe, D. D., A. Hiller, R. A. Phillips, P. B. Hamilton, V. P. Dole, R. M. ArChibaId, H. A. Eder, J. biol. Chemistry 183, 331 (1950). 11. Rerss, E., Dissertation Straßburg (1902). - 12. REIss, E., 
Erg. inn. Med. 10, 531 (1913). - 13. AlDER, A. Handbuch der norm. und pathol. Physiologie von A. Bethe und G. von Bergmann, Bd. VI, 1. Hälfte, S. 534, Berlin (1928). - 14. LoEwe, F., Optische Messungen des Chemikers und des Mediziners. 5. Aufl., Th. Steinkopff, Dresden und Leipzig (1949). - 15. Rrva, G., Das Serumeiweißbild. S. 176ff., 1. Aufl., Huber, Bern-Stuttgart (1957). - 16. Weber, E., Grundriß der biologischen Statistik, 5. Aufl.,
VEB G. Fischer Verlag, Jena (1964). - 17. Doerfel, K., Statistik in der analytischen Chemie, 1. Aufl., VEB Deutscher Verlag für Grundstoffindustrie, Leipzig (1966). - 18. Wissenschaftliche Tabellen, Documenta Geigy, 6. Aufl., Basel (1960). - 19. Heusser, H. und J. Scrineider, Schweiz. Med. Wschr. 77, 1327 (1947).
Priv. Doz. Dr. Dr. D. Stamm 8 München 23

Kraepelinstr. 10

\title{
Some Abnormal Permeability Characteristics of Ehrlich Ascites Tumor Cells ')
}

\author{
By L. A. KIEsow \\ Division of Molecular Energetics, Bioenergetics Laboratories, Naval Medical Research Institute, Bethesda, Maryland 20014, \\ U. S. A.
}

(Eingegangen am 11. April 1968)

Dedicated to Ernst Sabiitte on the occasion of bis 60 th birtbday

The intracellular and extracellular distribution of inorganic phosphate was studied with Ehrlich ascites tumor cells and trypsinized kidney cortex cells. Both, the intracellular and also the extracellular concentrations of inorganic phosphate were varied experimentally. The reported data suggests abnormal permeability characteristics of the Ehrlich ascites tumor cell.

Die intra- und extrazelluläre Verteilung von anorganischem Phosphat wurde an Ehrlich-Ascites-Tumorzellen und trypsinierten Nierenrindenzellen untersucht. Sowohl intra- als auch extrazelluläre Phosphatkonzentration wurden im Versuch variiert. Die Ergebnisse zeigen ein anormales Permeabilitätsverhalten der Ehrlich-Ascites-Tumorzelle.

There is little doubt that to establish permeability barriers is one of the foremost. functions of the cell membrane. To provide the membrane with such capabilities, the chemical nature of the permeating molecule and the structural and chemical nature of the cell membrane are acting in concert thereby permitting the cell to maintain a controlled interior milieu. It is furthermore well-known that a number of cellular enzymes support the membrane in this performance. Such is, for example, achieved by the intracellular introduction of a charged group into a previously uncharged molecule whereby it is rendered greatly impermeable after it entered the cell.

Nevertheless, exceptions to this general function of cell membranes have been described in the past. It was shown that even enzymatically active proteins, like aldolase (EC 4.1.2.13) may be released by certain cells $(1,2)$. Moreoxer, molecules like $\mathrm{NADH}$ were demonstrated to effectively interact on the site of intracellular enzymes when added extracellularly and to thereby influence metabolic rates like the rate of glycolysis (3). More recently the lack of membrane permeability barriers of certain intracellular parasites was shown to be one of their most outstanding features permitting these organisms to easily utilize metabolites, energy donors, and coenzymes provided by the host cell (4). This is a striking example, indeed, since it indicates how permeability characteristics of cellular membranes may gain control over metabolic patterns

1) The experiments reported herein had been concluded by December 1961 at the Physiologisch-Chemisches Institut der Freien Universität Berlin. and metabolic rates of both the intracellular parasite and the host cell as well. This relationship between a parasite and its host is mainly caused by the mutual exchange of metabolites and metabolic end products and it affects the metabolism of both the parasite and the host cell quite drastically. Such a relationship may not only permit the growth of the parasite but it may also destroy the host cell at the same time.

It becomes therefore quite apparent that the peculiarities of cell membranes safeguard an individual cell, thus permitting it to maintain distinct intracellular conditions. If, however, the membrane fails in this function or if some membrane characteristics are altered in a way that it no longer can perform this task, it inevitably exposes the cell's interior to influences of the exterior environment. Hence, any cell with a failing membrane will more or less have to adapt to or will be affected by the metabolite concentration and the ionic composition of the extracellular fluid prevailing in the organ in which this event occurs.

Some unusual features which may indicate abnormal permeability characteristics of the cell membrane of Ehrlich ascites tumor cells shall be reported in this paper. The experiments described herein pertain primarily to the permeation of the inorganic phosphate ion. They were executed mainly for three reasons:

(1) the permeation of phosphate ions will necessarily show many phenomena common to all such processes that involve ionized molecules or ions per se.

(2) the inorganic phosphate ion is known to exert considerable metabolic control within the cell involving individual enzymes and consequently metabolic pathways like glycolysis $(5,6)$ and cell respiration $(7)$. 